\title{
Application of Optical Fiber Sensing Technology in Oil Tank Level
}

\author{
Measurement \\ Chong-yong SU* and Shu-ren YANG \\ Northeast Petroleum University, Daqing, Heilongjiang, China \\ ${ }^{*}$ Corresponding author
}

Keywords: Optical fiber, Liquid level instrument, Measure

Abstract. Fiber optic sensing is a high-tech technology that has been developed since the late 1970s when fiber was successfully used in communications. Once it comes out, to the intrinsically safe explosion-proof, high precision, long-distance transmission, not afraid of corrosion, anti-electromagnetic interference and other unique advantages won the favor of the people In the fields of petroleum, chemical industry, nuclear industry and other areas of some traditional sensors inconvenient applications, can be accurate and convenient for a variety of physical measurement.

\section{Introduction}

With the development of petrochemical industry, oil tank automatic measurement technology has been widespread concern ${ }^{[1,2]}$. Especially in the past 10 years, due to the rapid development of high-tech technology such as computers, microelectronics, optical fibers and sensors, a variety of new technologies, new methods have been infiltrated into the field of tank metering. This paper introduces the application of optical fiber sensing technology in Oil Tank Level Measurement.

\section{Measurement Principle of Optical Fiber Liquid Level Meter}

\section{Overall System Description}

Optical fiber liquid level meter is divided into two kinds of normal pressure and pressure type. They are the use of force balance principle to achieve liquid level detection. The difference is that the normal pressure type is directly connected with the float by the steel wire rope, and the pressure type is driven by the magnetic coupling system to drive the upper and lower movement of the magnetic hammer. The mechanical inspection system is completely isolated from the optical coding encoder chamber. The following focuses on the working principle with pressure. The system consists of measuring unit (including floating ball, heavy hammer, guide wheel, sheave, magnetic coupler and other mechanical parts), optical fiber sensor, photoelectric converter, secondary instrument and cable. The working principle is shown below: 
Flammable and explosive dangerous places



Figure 1.Overall schematic optical fiber liquid level meter

Through the floating ball (or magnetic floating ball system) will be measured liquid level signal into the precise rotation of the measuring wheel, and the optical encoder of the optical fiber sensor is driven, so that the level of movement every $1 \mathrm{~mm}$ will produce a pulse signal, and then by quadratic table magnification shaping, reversible judgment and other processing shows the liquid level, at the same time, the output standard signal by RS2232C standard computer interface into the computer terminal management system ${ }^{[3,4]}$.

The whole system consists of five parts. Magnetic coupling detection system and optical fiber sensor installed in two parts of the flammable and explosive work site: The photoelectric conversion system, the secondary instrument and the computer system are installed in a safe control room. The transfer of liquid level information between the field and the control room is connected by the optical cable, do not tank electrical detection, safety explosion-proof. The following describes the various parts of the working principle and structure. The figure below shows the installation diagram of the optical fiber liquid level meter:

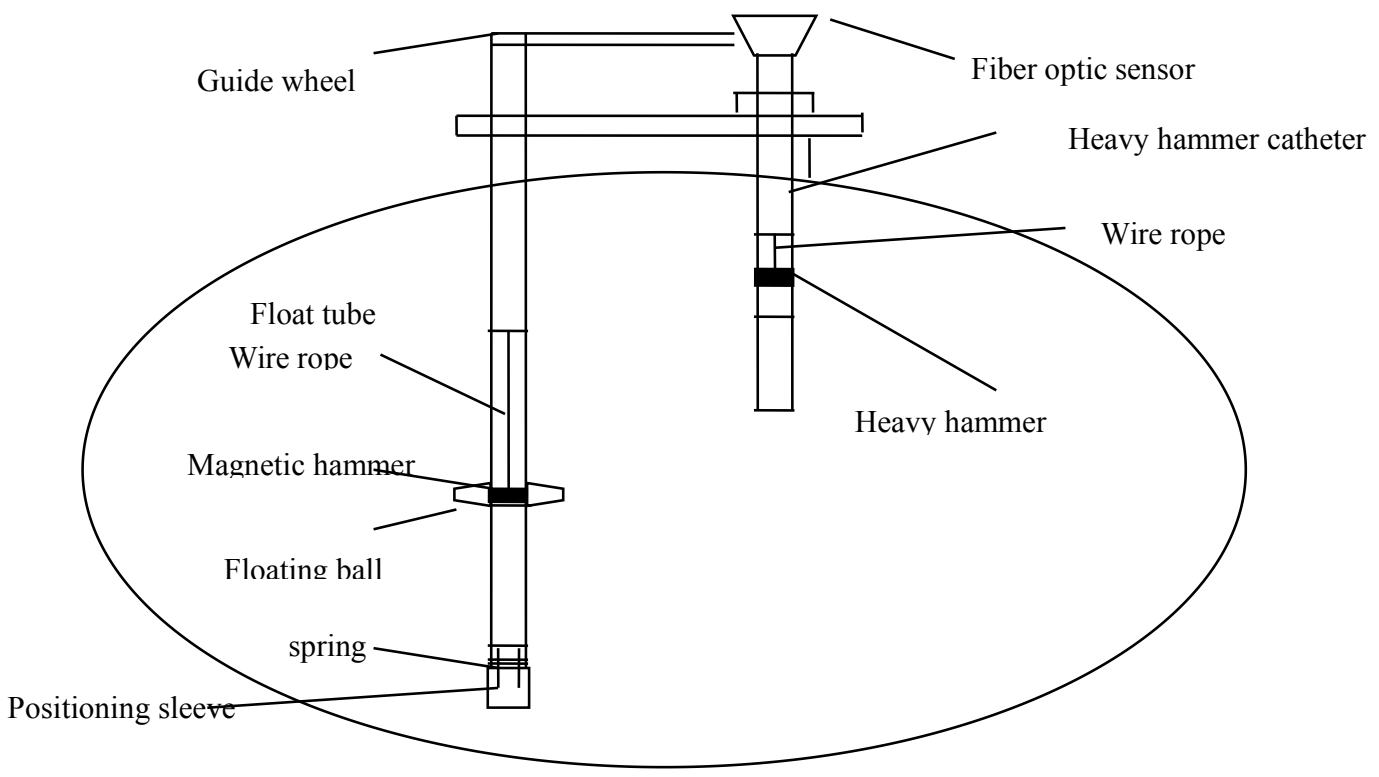

Figure 2.Installation diagram of optical fiber level meter 


\section{Magnetic Coupling Detection System}

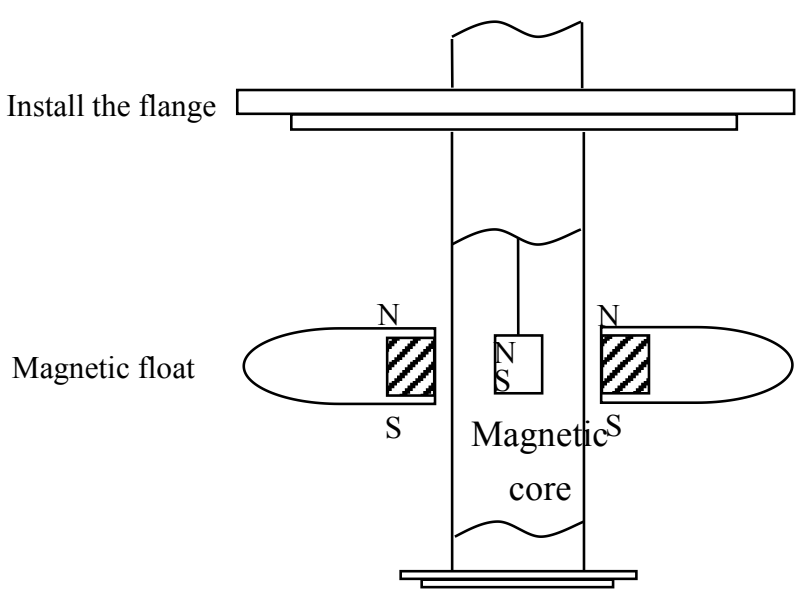

Figure 3.Structure of magnetic coupling detection system

The magnetic floating ball which tracks the change of the liquid level is driven by the interaction of the magnetic hammer in the pressure isolating pipe and the pulley of the magnetic core rope, The upper and lower movement of the liquid level is converted into the rotation of the optical encoder disc, and the angle and direction of the rotation of the optical encoder are measured, and the height of the liquid level in the spherical tank can be determined.

\section{Optical Fiber Sensing System}

The optical fiber sensing system is mainly composed of a magnetic wheel, an optical encoder, a reel and an optical fiber probe. The optical code disc is a thin plate with information code along the circumference. The material is $1 \mathrm{Cr} 18 \mathrm{Ni} 9 \mathrm{Ti}$, which is coaxial with the magnet and sealed in the disc chamber, completely isolated from the environment.

\section{Photoelectric Conversion and Secondary Instrument Parts}

Photoelectric converter and the scene of the fiber level gauge by the four-core armored cable connected. Two of the core to use the infrared light-emitting diode LED two sets of photoelectric converter light source. The probe sends a steady continuous light. The two sets of photoelectric conversion device will be another two-core optical fiber signal into electrical signals, and the signal processing is carried out in the special shielding box, and converted to a standard CMOS voltage signal to the secondary instrument.

After the photoelectric conversion and plastic amplification processing, it can come into the secondary instrument after the liquid level signal, in the secondary table after processing into the microcontroller. In the microcontroller, it will complete the reverse logic processing and other functions. The liquid level is displayed by LED. At the same time, the signal can be sent to the host computer through the standard RS2232C interface, and conduct tank farm management and monitoring.

\section{The Main Technical Indexes of Optical Fiber Liquid Level Meter}

Measuring range: $0-15 \mathrm{~m}$ 
Measurement accuracy: $\pm 2 \mathrm{~mm}$

Sensitivity: $1 \mathrm{~mm}$

Maximum transmission distance: $5 \mathrm{~km}$

\section{Optical Fiber Level Meter characteristics}

(1) Intrinsically safe explosion-proof. The liquid level meter is used in the field of optical signal, no electrical signal, so safe and reliable.

(2) Anti - electromagnetic interference. The optical signal is not subject to any electromagnetic interference in the transmission path.

(3) High precision and high sensitivity. It can fully meet the requirements of the application level of not strict requirements.

(4) Less moving parts, reliable operation, easy to use. In addition to floating roof, the measurement is not affected by bad weather such as 5 above the wind, rainfall, snow and other effects.

(5) Strong corrosion resistance. The main components of the field instrument is using a large number of stainless steel, PTFE self-lubricating bearings and other materials, without maintenance, and sealing performance.

(6) Easy installation. For the old tank transformation, do not need to fire on the tank openings, can use the original steel table with 3 holes in the 2, can also be installed in the top of the hole on the hole.

\section{Conclusion}

Optical fiber sensor from crude oil, light oil to gas, from the external floating roof, internal floating roof tank to vault are applied.Fiber optic sensor measuring oil tank liquid level height,and sents a high accuracy of oil tank level measurement system. It can realize oil tank liquid level, volumetric display and real-time warning function, In essence to ensure that the tank tank level of non-real-time measurement.The system has the characteristics of intrinsically safe, automatic display of the liquid level and automatic warning.

\section{References}

[1] Murashkina T L. Fiber optic liquid level indicator Telecommunications and Radio Engineering[J].1995,49(12):46-48

[2]Kashyap R1 Photosensitive optical fibers: Devices and applications[J].1 Optic Fiber Technol,1994,1:17.

[3] Hill K Omelets G. Fiber brag grating technology fundamen2tal and overview [J].Light ware Technol,1997,15 (8):12632-12761.

[4] Morey W , Meltz G, and Glenn W H. Fiber Bragg grating sensors[C].1 In Proc SPIE Fiber Optic \& Laser Sensors VII,1989. 